REVISTA DE DERECHO UNED, núm. 6, 2010

\title{
D. RAFAEL GIBERT SÁNCHEZ DE LA VEGA. «HISTORIA DEL DERECHO EN LA DISTANCIA» IN MEMORIAM
}

\author{
REMEdIOS MORÁN MARTín \\ Catedrática acreditada de Historia del Derecho y de las Instituciones \\ UNED \\ rmoran@der.uned.es
}

De esta manera, «Historia del Derecho en la Distancia» ${ }^{1}$, titulaba Rafael Gibert un trabajo en homenaje a José Martínez Gijón, también catedrático de Historia del Derecho en la Universidad de Sevilla, en el cual publicaba tres guiones emitidos por radio durante el periodo en el que él ocupó la cátedra de Historia del Derecho de la UNED.

El 22 de abril pasado nos dejó Rafael Gibert, en la festividad de San Agapito, papa, como a él le gustaba señalar en cada una de sus fechas. Sirvan estas páginas como homenaje a su memoria, partiendo de la metodología en la enseñanza a distancia que él inició en esta asignatura en nuestra Universidad.

Rafael Gibert (nació en Madrid, el 5 de julio de 1919) fue el primer catedrático de Historia del Derecho de la UNED, desde el año 1973 al 1984, por lo tanto desde un año después de la creación de nuestra Universidad. Diez años en la larga vida del profesor que lo marcaron de forma especial, porque nunca dejó de tener contacto asiduo con la Facultad, de diferente forma: eran habituales sus visitas y sus colaboraciones hasta los últimos años de su vida, así como la relación personal con alguno de los profesores del Departamento, hasta que por motivos de salud, se fue dilatando su presencia. Tampoco

1 Rafael Gibert, «Historia del Derecho en la distancia», en Historia. Instituciones. Documentos, n. ${ }^{\circ} 11,1984$, pp. 15-36. 
dejó de mantener relación con otros Departamentos de diferentes Universidades, como especialmente la Universidad Rey Juan Carlos, la Universidad Complutense (de la que fue profesor gran parte de su carrera universitaria) y el CEU, porque por encima de todo se sintió siempre un universitario.

En estas páginas de recuerdo a su figura, quisiera hacer un breve recorrido por su trayectoria, pero no lo voy a hacer como puede ser esperable en este momento, con una biografía más o menos amplia (que desarrollaré en otro lugar y que tengo en elaboración, por la importancia de su aportación a la Historia del Derecho), sino que ahora quisiera poner de relieve algunas de las actividades que realizó en esta Facultad de Derecho de la UNED, abriendo camino a muchas de las iniciativas que fueron y son específicas de la enseñanza a Distancia.

Inició su carrera profesional en el cuerpo técnico del Ayuntamiento de Madrid, como letrado (1945-1950), motivo por el cual muchas de sus publicaciones versaron sobre las instituciones madrileñas o que se desarrollaron en su espacio, como el Concejo de $\mathrm{Madrid}^{2}$ o El Consejo del reino ${ }^{3}$. Pasó directamente de auxiliar interino en la cátedra de D. Galo Sánchez su maestro, en la Universidad Central, a Catedrático en 1950, obteniendo el número uno de esta oposición y ocupando la cátedra de Granada, donde fue Secretario General. Tras acceder a la cátedra siguió completando su formación en Bonn y en Roma entre 1950 y 1954, donde fue vicedirector del Instituto Hispánico Jurídico de Roma, siendo director en aquel momento Álvaro D'Ors.

Ocupó la cátedra de Granada hasta el año 1971, cuando por concurso de traslado volvió a la Universidad Complutense de Madrid, en la que también ocupó los puestos de Vicedecano de la Facultad de Derecho, siendo decanos Juan del Rosal, Luis Legaz Lacambra y Alfonso García de Valdecasas. El profesor Gibert también fue Decano en funciones, primero tras la dimisión de Juan del Rosal y posteriormente de Alfonso García de Valdecasas.

En 1979 fue nombrado miembro de la Academia Portuguesa da Història. Se jubiló en la Universidad Complutense de Madrid a los 68 años, en 1987, lo cual significó para él un duro golpe. Sin embargo, prácticamente hasta los últimos años de su vida ha seguido escribiendo y participando en numerosos actos académicos.

${ }^{2}$ El Concejo de Madrid. Su organización en los siglos XII a XV, Gráficas Martínez, Madrid, 1949.

3 El antiguo Consejo de Castilla, RIALP, Madrid, 1964. 
Su presencia en la UNED, tanto en la Facultad de Derecho como en el Curso de acceso para mayores de 25 años, se desarrolló desde 1973 a 1983, a propuesta del entonces rector Manuel Jesús García Garrido, compatibilizando inicialmente su actividad docente con la Universidad Complutense y siguiendo los criterios iniciales del entonces Ministro de Educación, Julio Rodríguez Martínez, de que los profesores de la UNED fueran los que ocupaban puestos en otras universidades. Así, fue el encargado de la cátedra de Historia del Derecho, siendo en aquel momento profesores en la misma Ramón Fernández Espinar (que fue también Secretario general de la UNED) y Rafael Zurita Cuenca.

Asimismo desde 1974 hasta su vuelta a la Universidad Complutense fue Decano del Curso de acceso para mayores de 25 años. Fueron muy conocidas sus «Reuniones de los jueves», que cada semana reunía tanto a los profesores como al personal de administración y servicios, para tratar los problemas del curso de acceso y a partir de las cuales se constituían Comisiones para resolver los temas concretos que se planteaban.

De la mano del profesor Gibert proceden las primeras Unidades didácticas, Introducción al Derecho ${ }^{4}$, para la asignatura del mismo nombre del Curso de acceso de mayores de 25 años.

Captó Rafael Gibert la metodología de la enseñanza a distancia, como puede apreciarse si se leen las Unidades didácticas, en las cuales, en la edición primera, de 1976, pasados los primeros tres cursos, tiene para mí el acierto de introducirlas con la edición de lo que él llama «Preliminares del curso 1973», y después de 1974, se va apreciando el desenvolvimiento de la metodología que aplica en su docencia:

«Exige el ritmo de nuestra Universidad, que la materia de este curso se distribuya en tres Unidades Didácticas, y cada una conste de seis Temas, los cuales a su vez han de ofrecer al alumno: un esquema del mismo, una instrucción sobre su estudio, un ejercicio de autocomprobación, y una pequeña serie de actividades que se recomiendan para alegrar su espíritu, para impulsarle a la superación, para que no se sienta constreñido por una disciplina que quiere darle libertad $»^{5}$.

Fiel a las primeras indicaciones sobre la puesta en marcha de la UNED, Rafael Gibert en este «Preliminar» de 1973 a la Introducción

\footnotetext{
${ }^{4}$ UNED-Ministerio de Educación y Ciencia, Madrid, 1976. Existen ediciones hasta 1983, posiblemente no de todas las Unidades didácticas.

5 Preliminar del curso 1973, Ibid., s.p.
} 
al Derecho, recomienda el libro de Álvaro D’Ors, Una Introducción al Estudio del Derecho ${ }^{6}$. Al hacerlo se dirige a los alumnos, como si de una carta se tratara, en un tono directo y cercano, presentando al autor del libro, su obra y relacionando ésta con su propio concepto de Derecho, como un estudio de «libros», recomendando éste, pero sin excluir los demás. Y, en lo que puede decirse que se trata de una exposición de los objetivos, va enumerando las partes de que se compone, lo que el alumno va a encontrar en sus páginas y las enseñanzas que va a poder recibir, exponiendo, además la forma de resolver los ejercicios de autoevaluación en la que es absolutamente magistral su manera de introducir al alumno en la posibilidad de diferentes respuestas, advirtiendo que se debe a diferentes concepciones del Derecho:

«Cuando responda a las preguntas no debe interesar al alumno si acierta o no la respuesta. Lo importante es que él perciba la pregunta, y examine las respuestas. Acaso a él se le ocurra una cuarta o quinta respuesta posible. Apúntela. Después elija. Es como un juego. Elija aquélla que le parezca más exacta, o bien la única exacta, después de haber rechazado las otras dos, como inexactas, no verdaderas, o simplemente como menos ciertas, o como secundarias. De todo esto hay. A veces, las tres respuestas contienen aspectos diferentes, todos válidos; entonces es preciso, para acertar, que se dé cuenta de cuál es la más oportuna, por el contexto, la intención, la línea de pensamiento que seguimos. Pero no se deprima por "no acertar" itambién el alumno tiene sus razones! Y, a veces, la razón. Es muy posible que el alumno no sea un ignorante de cuestiones jurídicas. Que tenga formada su opinión o la vaya formando. Y en éste o aquel punto se encuentre en posición contraria a la del autor del libro que seguimos. Entonces jalegría! Esto es la Universidad. El derecho encierra una dialéctica en la que es lícito participar».

Sigue más adelante sobre la forma de contestar a las preguntas del examen escrito, que será el medio de evaluación:

«No es necesario que respondan ni con las palabras, ni siquiera con las ideas, ni en la dirección que les marca el libro (o bien las instrucciones, o las respuestas a los ejercicios de autocomprobación), sino que basta con que el alumno ofrezca una respuesta congruente, también si está tomada de otros libros, de su propia experiencia o de su sentido natural de las cosas. Nuestra doctrina no es obligatoria; puede también adoptarse discrecionalmente; la madurez del alumno, y las ideas ambientales, suscitarán reparos. Lo importante es que el alumno aprenda que el autor dice esto o lo otro, y que él, sin embargo, prefiere esto o aquello. Con libertad, con responsabilidad que incluye con-

${ }^{6}$ Ediciones RIALP, Madrid, 1963. 
secuencia con las propias ideas, y también registrar las lagunas, las dudas, las contradicciones, que son la carga de todo conocimiento en esta tierra ${ }^{7}$.

¡Qué actual resulta esta metodología! Parece que leamos las directrices para la adaptación de los materiales didácticos al Espacio Europeo de Educación Superior.

Según él mismo manifestó con frecuencia, fue un entusiasta de la enseñanza a distancia y lo mostró en dos aspectos fundamentalmente: en la admiración por los alumnos que estudiaban a distancia ${ }^{8}$, reconociendo el esfuerzo que realizaban y que se apreció de una forma muy especial en su actuación en el Curso acceso para mayores de 25 años ${ }^{9}$, en el que trabajó con verdadera vocación y en las diferentes formas de comunicación que la distancia proporcionaba para la docencia. Las palabras transcritas son buena muestra de ello, al denotar una sensibilidad hacia esta forma de enseñanza que difícilmente se entiende si no es un verdadero profesor.

Rafael Gibert fue redactando desde el primer curso los materiales complementarios, que primero se enviaban en cicloestil y se imprimieron, completados a lo largo de los cuatro primeros cursos, en 1976 (que es la edición que consulto) y que consistían, como el profesor explica en los preliminares, para cada tema en: un temario, un esquema, instrucciones para el estudio, explicaciones complementarias, ejercicios de autocomprobación, soluciones a estos ejercicios y actividades recomendadas.

En segundo lugar, si interesante fue la experiencia que nos traslada su docencia en "Introducción al Derecho», no menos lo fue la que desarrolló en el entonces Departamento de Historia y Filosofía

7 Ibid., s. p.

8 Rafael Gibert, «Historia del Derecho en la distancia», o. c., p. 16. Recomiendo la lectura de la segunda parte de este trabajo, «Diálogo sobre concepto y plan», emisión de radio hecha a modo de diálogo entre profesor, una alumna y un alumno repetidor, lleno de humor, ironía y a la vez de respeto hacia el alumno, en el que intenta una provocación, especialmente al final.

9 «Si a un servidor le preguntaran, y se lo han preguntado, cuáles son las razones que le han decidido a abandonar la Universidad convencional, y concretamente la de Madrid, llamada Complutense, en la que ha sido sumamente dichoso durante estos diez años, la respuesta sería que una de las razones, no la única, pero sí positiva y real, ha sido la de encontrar nuevamente y continuar junto a ellos, a un número de alumnos con los cuales se siente en íntimo contacto a través del curso de Introducción al Derecho, dictado a los mayores de veinticinco años que acceden directamente a la Universidad, una comunidad escolar a la que presumo conocer y en todo caso estimo grandemente», Ibid., p. 19. Se trata de la publicación del guión de una emisión de radio, titulada «Primer apoyo-radio del curso 81-82». 
Jurídicas, cátedra de Historia del Derecho, donde desarrolló una serie de iniciativas, adaptadas a la enseñanza a distancia que considero importante poner de relieve: la coordinación y participación en las Unidades Didácticas, la elaboración de un libro pionero de lo que sería la autoevaluación de los alumnos a distancia, la grabación de emisiones de radio, la redacción de sus famosos «correos académicos» y, finalmente, en materia de investigación, la celebración de la VI Semana de Historia del Derecho.

Si en la asignatura de «Introducción al Derecho» para el Curso de acceso de mayores de 25 años elaboró las Unidades didácticas, según he expuesto, en «Historia del Derecho español», por el contrario, ejerció una función de coordinador de los materiales.

En Historia del Derecho, desde el año 1973, el profesor Gibert recomendó para el estudio de la asignatura su libro Historia General del Derecho Español ${ }^{10}$. Siguiendo las indicaciones generales, las Unidades Didácticas para las diferentes asignaturas de las Licenciaturas, se debían o bien elaborar como nueva edición, o bien adaptar el manual recomendado a la metodología de la enseñanza a distancia. En Historia del Derecho se elaboraron, bajo la dirección del profesor Gibert, estas Unidades didácticas, que inicialmente también se mandaban mecanografiadas y cicloestiladas en forma de cuadernillos, dividiendo el curso en 6 Unidades didácticas, cada una de seis temas ${ }^{11}$. Ya en el programa, que difería muy sustancialmente de la Historia General del Derecho, y en estas Unidades didácticas, se inicia la costumbre muy consolidada en la UNED hasta fechas recientes, de dividir los programas de todas las asignaturas en 36 temas, 18 para cada una de las pruebas presenciales.

Siguiendo su lectura y aunque el mismo profesor Gibert decía que todo se hacía colegiadamente entre los tres miembros del equipo

\footnotetext{
${ }^{10}$ Cuya primera edición la había publicada en Granada, F. Román, 1968, manual del que hizo una nueva edición en Madrid, Realigraf, 1971 y otra coincidiendo con su llegada a la UNED, en 1973. Hay ediciones posteriores, Madrid, Copigraf, 1974 y 1977; Madrid, M. Huerta, 1981.

11 Fueron redactadas por Ramón Fernández Espinar y Rafael Zurita Cuenca, bajo la dirección de Rafael Gibert. La primera edición que tenemos es de 1974, se conservan en formato mecanografiado y cicloestilado, encuadernado en un volumen, UNED-Ministerio de Educación, Madrid, 1974 y las Unidades didácticas, Historia del Derecho, Universidad Nacional de Educación a Distancia, UNED-Ministerio de Educación, Madrid, 1975. Por catálogos, puede seguirse que se hicieron ediciones hasta 1981, con lo que desde estas páginas no puedo sino lamentar que no se haya conservado ni siquiera en la Biblioteca de la UNED un ejemplar del material recomendado en la asignatura de cada uno de los cursos.
} 
docente $^{12}$, creo que se puede fácilmente diferenciar lo que pertenece a su autoría y lo que pertenece a los demás profesores que compartían la docencia; en otro lugar especifica que «Se han reproducido y repartido ya las Unidades que fueron redactadas por un servidor en 1973, con la colaboración de los doctores Fernández Espinar y Zurita Cuenca (...). Al primero debo agradecer, con otros beneficios, el de haber conservado y enriquecido con Addendas nuestras comunes Unidades ${ }^{13}$. Sin embargo, se observa que fueron publicadas para completar y adaptar la Historia general del Derecho español, y aunque no aparece diferenciada la autoría de las diversas partes que componen dichas Unidades, tanto por el estilo como por publicaciones posteriores ${ }^{14}$. Por su parte, solo dos cursos después de las primeras Unidades didácticas, el profesor Gibert, escribe que la publicación de su libro Elementos formativos del Derecho en Europa ${ }^{15}$, responde a la necesidad de introducir el primer tema referente al concepto y método que no incluía su Historia General, y que hasta el momento se había respondido al programa de la UNED mediante la Addenda de la primera unidad didáctica ${ }^{16}$.

Los ejercicios de autocomprobación y sus respuestas con seguridad proceden de Rafael Gibert, no solo por seguir la misma técnica y estilo que los ejercicios recogidos en las Unidades didácticas de Introducción al Derecho, ya comentadas y de su total autoría, sino porque años después, cuando redacta un libro único en la experiencia de la Facultad de Derecho de la UNED, 360 preguntas y respuestas sobre Historia del Derecho español, él mismo se refiere a los ejercicios de autocomprobación de las Unidades didácticas, considerando que ha

12 Rafael Gibert, «Historia del Derecho en la distancia», o. c., p. 19. En las pp. 20 a 28 hace una completa exposición de la metodología a distancia, hablando de modo directo a los alumnos, como de una emisión de radio que es. Esta aportación de Rafael Gibert es digna de lectura, especialmente para los profesores jóvenes de la UNED.

13 Ibid., 35

14 Se puede saber que principalmente la adaptación inicial se debe a Ramón Fernández Espinar, puesto que gran parte de ellas, lo referido a las explicaciones preliminares de cada tema y los esquemas (salvo los que reproduce de otros manuales, como él reconoce), fueron posteriormente publicadas por él en Las fuentes del Derecho histórico español. Esquema y resúmenes, Centro de Estudios Universitarios Ramón Areces, Madrid, 1985. También la parte primera de metodología en Id., Antología de textos jurídicos históricos, con orientaciones metodológicas para su comentario, Granada, 1990. Véase, asimismo, Id., «La enseñanza del Derecho», en BFD, n. ${ }^{\circ}$ 6-7, 1981, pp. 93-97.

15 Madrid, 1975 (se hizo una segunda edición en 1982, con las adiciones que el autor señala en el preliminar).

16 Además del «Preliminar» a las dos ediciones de este libro, expone este mismo dato en «Historia del Derecho en la distancia», o. c., p. 36. 
llegado el momento de ampliar aquellos ejercicios de autoevaluación y considerando las respuestas no solo como verdaderas o falsas, sino como ampliación de las explicaciones recogidas en aquéllas, por lo que en mayúscula, las subtitula Addenda para el curso primero en la Distancia ${ }^{17}$.

Por otro lado, en las respuestas que va dando Rafael Gibert al final de las 360 preguntas se puede ver una amplia gama de su faceta docente (pp. 81-225): su estilo directo con los alumnos, sus recuerdos de vivencias en las «convivencias» en Centros asociados, sus explicaciones adicionales entre teoría y práctica, la provocación que a veces intenta con los alumnos, etc. Véase especialmente las pp. 115-116, en las cuales explica al alumno, como si de una conversación en directo se tratara, de cómo se hace un comentario de texto, en esencia como una reflexión personal del alumno. Leer estas respuestas del profesor Gibert es todo un ejercicio de un profesor, que siempre lo fue presencial y que se ilusionó por la distancia.

En estas respuestas se habla con frecuencia de los Cuadernillos de evaluación a distancia ${ }^{18}$, preceptivos en aquel momento, pero tengo que decir que no he podido consultar ninguno, no sé si alguno de ellos aún se conserva en los Centros Asociados. El mismo Rafael Gibert, en el Boletín de la Facultad de Derecho, hace referencia a los mismos, suprimiendo algunas de las pruebas ${ }^{19}$, facilitando las respues$\operatorname{tas}^{20}$, etc.

Pero él no solo se dejó llevar por las «directrices oficiales», desarrolló una gran imaginación para acercarse a los alumnos y a los tutores. A los primeros se dirigió no solo por los medios habituales de emisiones de radio, ejercicios de autocomprobación, dirección de las Unidades didácticas, Addendae a las mismas, en el Boletín de la Facultad de Derecho de la UNED (primera época) ${ }^{21}$ o convivencias, sino que en cualquiera de los momentos aprovechaba una publicación, como en el caso de las referidas 360 preguntas y respuestas, para al fi-

\footnotetext{
17 Universidad Nacional de Educación a Distancia, UNED, 1982.

18 Lo que posteriormente conocemos como Pruebas de evaluación a distancia (PEDs).

19 «Historia del Derecho», BFD, n. ${ }^{\circ}$ 5, 1980, p. 7.

20 «Historia del Derecho español. Respuestas del catedrático al primer cuadernillo del curso 1982-1983», en BFD, n. ${ }^{\circ}$ 11-12, 1984, pp. 85-105. Aunque este número del Boletín se publicó en 1984, se refiere al curso 1982-1983, último de los que estuvo el profesor Rafael Gibert en la UNED, por lo que es de suponer se envió a los tutores fotocopiados en el curso correspondiente antes de aparecer impresos.

${ }^{21}$ Véase su larga Addenda a alguno de los temas, en $B F D$, n. ${ }^{\circ} 11-12,1984$, pp. 107120. Repito lo mismo que en la nota anterior sobre la fecha de publicación.
} 
nal, incluir un epílogo, en el cual comentaba una de las preguntas del examen, en este caso sobre el Fuero de León y después del epílogo, aún introduce, seguro que en últimas pruebas, lo que llamó «Buzón de alcance», en el que no solo comenta las reclamaciones de los alumnos a los exámenes, sino que propone un ejercicio para la elaboración de un índice de la Addenda, incluyendo una ficha, y que será valorado como mérito $^{22}$. Termina con unas Observaciones del alumno. Visto desde la perspectiva de los nuevos Grados, fue un verdadero pionero.

También valoró públicamente la labor de los profesores tutores. En varias de sus publicaciones agradeció su labor y nombró, nominalmente, a los profesores tutores de la asignatura. Si se lee la exposición que publicó tras el Seminario de profesores tutores de Historia del Derecho de 1981, en lo que puede considerarse un acta de aquella reunión, se apreciará el rigor y sentido que dio a aquellas reuniones ${ }^{23}$.

A diferencia de otros catedráticos, que «ocultan» su pertenencia a nuestra Universidad, el profesor Gibert siempre que tuvo oportunidad tanto en su década de estancia en ésta como posteriormente, llevó como bandera a esta Facultad y a la UNED. Valga su saludo en el coloquio sobre "La cultura ibérica y el derecho romano», celebrado en 1980 en la Universidad de Sassari (Cerdeña) ${ }^{24}$, o su artículo, ya citado varias veces, en el que de forma tan elocuente expone su experiencia en nuestra Universidad, "Historia del Derecho en la Distancia» ${ }^{25}$,

22 En el «Preliminar de 1982» a la edición de este año de su libro Elementos formativos del Derecho en Europa. Germánico, romano, canónico, agradece a los alumnos de la UNED que han hecho este trabajo de índice, p. X.

${ }^{23}$ Rafael Gibert, «Tutores de Historia del Derecho español», en $B F D$, n. ${ }^{\circ}$ 9-10, 1983, pp. 13-31. Entre las muchas menciones a los tutores, vid., «De la VI Semana de Historia del Derecho», en Historia. Instituciones. Documentos, 19, 1989, pp. 46 y 56.

24 «Y aprovecho la ocasión para consignar un afectuoso saludo a nuestra Facultad en la Distancia que encuentro floreciente, cuando me reincorporo como titular, tras haberme encargado de la Asignatura en su momento inicial, en 1972», Rafael Gibert, «El "Nuevo Savigny" en España», en BFD, n. ${ }^{\circ}$ 8, 1982, pp. 25-34.

25 Se trata de su colaboración al homenaje a José Martínez Gijón y en él reproduce, en primer lugar, lo que denomina «Método de enseñanza», que dice ser un «texto redactado al promediar el primer curso de la asignatura que me fue encomendado para 1973, como director de lo que en la Universidad a Distancia llaman el equipo docente... No puedo asegurar si un texto tan extenso fue grabado y emitido en su totalidad o en forma resumida. En todo caso es un documento relativo a esa Universidad, mal conocida, pienso; de sus aspiraciones y propósitos, y también de su efectividad. La segunda una piecesilla, un entremés, no la publico ahora sin cierto rubor, por sus pretensiones. Se trata de un guión de radio, éste sí efectivamente grabado... Está redactada a fines de 1974, para iniciar las emisiones de 1975. Por último, recojo el texto destinado a una primera emisión del curso 1981-82, incorporado el autor ya como propietario de la cátedra en la Universidad a Distancia, donde he cesado al volver a la llamada Complutense en Madrid y precisamente la que fue de don Galo Sánchez 
una vez que ha vuelto a la Universidad Complutense o, mucho más tarde, en el Homenaje al profesor Alfonso García-Gallo ${ }^{26}$. Nada más locuaz y expresivo que sus palabras al relatar, y en parte reproducir, su actividad docente en la UNED, en la cátedra de Historia del Derecho, sus emisiones de radio, en las Unidades didácticas, su relación con los alumnos, etc.

Tuvo un acierto más: la utilización frecuente de cartas, circulares y de lo que él llamó «correo académico». Álvaro D’Ors lo calificó como "glosas en cadena», augurando que, salvo la colección que pueda conservar el autor, será muy difícil encontrar su totalidad. Se refiere a los pequeños trabajos, glosas, sobre diferentes aspectos de Historia del Derecho y de los historiadores del Derecho, que publica Rafael Gibert en diferentes periódicos, especialmente en El Faro de Motril. Según aquél recoge, la primera vez que Rafael Gibert utiliza el rótulo de "Correo académico» es en El Faro de Motril, del 25 de noviembre de $1969^{27}$.

Pero, como característico del mismo, van a ser también los correos académicos que utiliza como medio de comunicación, de acercamiento de la distancia, entre catedrático y profesores tutores y entre aquél y los alumnos. Si difícil es recopilar los publicados en prensa, más difícil será la recopilación de los enviados en el seno de la UNED, a los que hace referencia en las respuestas de sus 360 preguntas y respuestas, en sus "Preliminares» a diferentes ediciones de sus libros, etc. y que a veces prodigaba regalar a colegas. Yo misma tengo algunos en los cuales informa de actividades, publicaciones, deficiencias y virtudes del sistema de enseñanza a Distancia, comentario sobre exámenes, convivencias, Seminarios, etc. siendo piezas hoy esenciales para el conocimiento de la trayectoria de la Historia del Derecho en los años de su permanencia en la UNED.

Finalmente, para terminar este recorrido del primer catedrático de Historia del Derecho en la UNED, deseo resaltar que Rafael Gibert no obvió la atención a la investigación, por lo que acometió en el seno

cuando se titulaba Central. Siéndome difícil recapitular tan interesante experiencia, me complace salvar estos fragmentos que algo dicen de ella», Rafael Gibert, "Historia del Derecho en la distancia», o. c., pp. 15-36.

26 "Alfonso García-Gallo y mi cátedra», en Homenaje al profesor Alfonso García-Gallo, Madrid, 1996, I, pp. 49, 57, passim. También reproducido en la página del Departamento de Historia del Derecho de la UCM, en «Maestros Complutenses de Historia del Derecho», http://www.ucm.es/info/hisdere/maestros/ggallo/rgibert.htm

27 Álvaro D’ Ors, «El “Correo académico” de don Rafael Gibert», en Historia. Instituciones. Documentos, n. ${ }^{\circ} 14,1987$, pp. 1-6. 
de su cátedra la organización de la «VI Semana de Historia del Derecho». De nuevo, sus referencias en diferentes trabajos, como en uno específico, a modo de cronista en este caso ideal, nos da luz sobre las «Semanas de Historia del Derecho» y sobre la dirigida y organizada por él ${ }^{28}$.

Como él mismo explica, las Semanas de Historia del Derecho se habían ido celebrando en diferentes Universidades. Se iniciaron en 1932 ó 1933, con la Primera Semana de Historia del Derecho. La Segunda, organizada por Alfonso García-Gallo, en 1948; la tercera, en Valencia, organizada por Juan García González; la cuarta en 1969 organizada por Ismael Sánchez Bella, en la Universidad de Navarra; la quinta en la Universidad del País Vasco, en San Sebastián, organizada por Gregorio Monreal; ahora, la sexta fue dirigida por el profesor Rafael Gibert en la UNED, en 1983, proponiendo un tema abierto, pero destinado al homenaje a los historiadores del Derecho vivos, que podría ser un análisis de su obra ${ }^{29}$.

No llegaron a publicarse las actas de dicha Semana, aunque algunos autores lo hicieron individualmente por lo que no nos ha llegado la cohesión que el organizador quería dar a la Semana. Sí consiguió la continuidad de las mismas y el inicio de la organización de Seminarios y Congresos en nuestra Facultad, que hasta ese momento no eran frecuentes.

Puede resultar extraño cómo llegó Rafael Gibert a sentir la enseñanza a distancia, a intentar siempre el acercamiento; a veces algunos profesores lo recuerdan aún como «el profesor de Historia que hacía al alumno pintar en el examen el perfil de su mano, en lugar de su firma». Yo he visto alguno de estos exámenes, aunque desgraciadamente no conservo ninguno. Un mucho de poesía en ello.

Escribía Rafael Gibert en el «Preliminar» de sus 360 preguntas y respuestas que «Al preparar estos ejercicios durante la vacación del verano, en la perspectiva de un nuevo curso, el catedrático ha tenido la sensación de que era vencida la distancia, nuestra condición radical y provechosa, y tiene la esperanza de que también los alumnos lleguen algún tiempo después a la proximidad de comunicación en que consiste siempre la Universidad». Seguidamente incluye unos «Le-

28 Rafael Gibert, «De la VI Semana de Historia del Derecho español (1983)», en Anuario de Estudios Medievales, n. ${ }^{\circ}$ 19, 1989, pp. 43-58. También se publica una reseña en $A H D E$, n. ${ }^{\circ}$ 53, 1983, pp. 704-712 e Id., "Notarios en la Historia del Derecho», en Revista de Derecho Notarial, n. ${ }^{\circ}$ 121-122, julio-diciembre, 1983, pp. 413-437.

${ }_{29}$ «De la VI Semana de Historia del Derecho español (1983)», o. c., pp. 43 y ss. 
mas» de poetas, acerca de la distancia y la cercanía, entre ellos, unos versos de Ora marítima, de Rafael Alberti:

Yo sé que lo lejano,

sí, que lo más lejano, aunque se llame...

no hace que los oídos

de tu siempre dispuesto corazón no me oigan.

Ahora, al recordar en estas páginas a Rafael Gibert, con el que siempre tuve una cercana lejanía, no puedo sino ver al profesor que fue, en su desubicación obligada de la Universidad, que nunca quiso abandonar, como el sentimiento de Alberti en su Marinero en tierra, su última lectura, su última recitación, su vuelta a su otra vocación: la poesía. 